\title{
Artificial Intelligence in Collaborative Computing
}

\author{
Xinheng Wang ${ }^{1} \cdot$ Honghao $\mathrm{GaO}^{2,3} \cdot$ Kaizhu Huang $^{4}$ \\ Accepted: 23 May 2021 / Published online: 10 November 2021 \\ (c) The Author(s), under exclusive licence to Springer Science+Business Media, LLC, part of Springer Nature 2021
}

Artificial Intelligence (AI) has been witnessed as one of the fastest growing enabling technologies to optimise the performance of various systems. For example, face recognitionbased security check can enable a secure and fast securitycontrol process at airports, Amazon Alexa can support the automation of our homes, and various customer services are supported by AI. These AI-enabled systems have paved a route for our society to moving to an era of smart lives. Recently, with the advancements of Internet of Things (IoT) and unmanned autonomous vehicles, the trend is moving forward at a faster speed. This new trend has also created even smarter systems with the collaboration of computing, interaction between virtual environment and physical world, and quality data-driven services. However, there are still a lot of technical challenges to be addressed to ensure the performance, reliability, security and privacy, and fair sharing of workload among various systems.

The nature of these IoT systems and unmanned autonomous vehicles is distributed. In order to effectively manage and operate these systems, collaborative computing is essential to ensure the quality of these systems. Collaborative computing with the support of $\mathrm{AI}$ is a very promising approach to address these challenges. The aim of this special issue is to explore recent advances in AI technologies in the collaborative computing field, which can address the theoretical and practical challenges for modern services and applications. Seven selected papers with high quality are featured in this special issue. These papers follow the latest technical development trends, and the key technical

Xinheng Wang

xinheng.wang@xjtlu.edu.cn

1 Department of Mechatronics and Robotics, Xi' an JiaotongLiverpool University (XJTLU), Suzhou 215123, China

2 School of Computer Engineering and Science, Shanghai University, Shanghai, China

3 Gachon University, Seongnam, South Korea

4 Department of Intelligent Science, Xi' an Jiaotong-Liverpool University (XJTLU), Suzhou, China challenges were addressed, ranging from the fundamental issues for the cyber-physical systems, to security protection for the IoT systems, server selection for mobile edge computing to support the applications of IoT systems, effective shopping recommendations with privacy concern embedded in the system, efficient route planning for swarm robotics, and demonstration of applications of collaborative computing in image processing.

In the first paper titled "Runtime Verification of SpatioTemporal Specification Language", a new specification language was proposed by combining signal temporal logic with a spatial logic to characterize spatio-temporal dynamic behaviours of cyber-physical systems. This language allows the description of quantitative signals by expressing spatiotemporal traces over real-valued signals in dense time, and Boolean signals by constraining values of spatial objects across threshold predicates. This work provides a valuable tool in designing and managing various types of cyber-physical systems.

IoT is an emerging paradigm to link various cyberphysical systems. However, security is always a concern to the public, particularly, to those who are using wearable medical devices to record health data and monitoring health conditions. In the second paper titled "Secure and Usable Handshake based Pairing for Wrist-Worn Smart Devices on Different Users", a secure handshake mechanism, which is widely used in pairing devices, was proposed by utilizing an optimal feature selection algorithm to improve the success rate and security of the system, and reduce the paring time to $<4 \mathrm{~s}$, which improves not only the security of the system, but also user experience.

With the wide use of IoT systems, mobile edge computing is the de facto tool to ensure real-time data processing and services provided by these IoT systems. However, users in the overlapped areas face the difficulty in selecting the right mobile edge server. In the third paper titled "Edge User Allocation in Overlap Areas for Mobile Edge Computing", the authors, firstly, have modelled this problem as a multiobjective optimization problem. Then, they have proposed a Pareto boundary search algorithm based on convex hull 
to reduce the complexity of widely used Pareto algorithm, which is effective but complex. Finally, a principal component analysis algorithm was designed to select the most suitable server.

IoT systems have been penetrating into every corner of human life. With the rich data generated from these IoT systems, the world is entering into a data-driven services, management, and manufacturing era. Shopping recommendation is one of the services to foster personalised shopping experience by using a large volume of data. However, data is not always there. Sometimes, data sparsity exists, particularly for new services. How to use the sparse data to provide accurate recommendation is a challenge. In the fourth paper titled "PR-RCUC: A POI Recommendation Model Using Region-Based Collaborative Filtering and User-Based Mobile Context", a new framework was proposed to address the challenge of data sparsity, where region factor and user preferences are considered to provide accurate recommendations. At the same time, the data could be misused, if the data is leaked. In the fifth paper titled "Point-of-Interest Recommendation with User's Privacy Preserving in an IoT Environment", a user's privacy preserving framework was proposed based on local differential privacy by integrating user's long-term static and time-varying preferences to protect the visited Points of Interest (POIs) and recommendation results to users, which ensures the privacy of the data whist providing services.

IoT systems are focusing on linking individual system into a networked world. Nowadays, with the development of unmanned vehicles, such as robots on the ground and drones in the air, cooperative action is a preferred choice towards accomplishing difficult missions. In this sixth paper, titled "Self-Organised Collision-Free Flocking Mechanism in Heterogeneous Robot Swarms", an extended model of a self-organised flocking mechanism using heterogeneous swarm systems was proposed by combining a collective motion mechanism with obstacle avoidance functions, which ensures a collision-free flocking trajectory for the followers.

Collaborative computing is not only applicable in addressing system challenges, but also addressing a specific challenge in technology. An application of collaborative computing in image processing was demonstrated in the last paper, titled "Fast Blind Deblurring of QR Code Images Based on Adaptive Scale Control". A fast blind deblurring algorithm for QR code images was proposed in this paper to solve the out-of-focus caused by lens shake, inaccurate focus, and optical noise. The proposed algorithm optimises productivity under the guidance of collaborative computing and achieves the effect of adaptive scale control by introducing an evaluation mechanism.
Acknowledgements The guest editors are thankful to our reviewers for their efforts in reviewing the manuscripts. We would like to sincerely thank the Edit-in-Chief, Dr. Imrich Chlamtac, for his supportive guidance during the entire process. We would also like to thank Jed Joseph Adel, Eliska Vlckova, and Leonora Panday for their continuous support in editing this special issue.

Publisher's Note Springer Nature remains neutral with regard to jurisdictional claims in published maps and institutional affiliations.

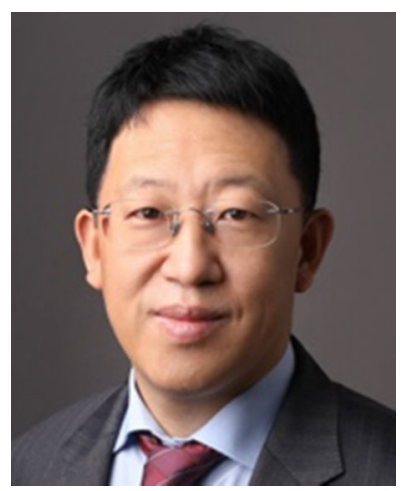

Xinheng Wang received the B.E. and M.Sc. degrees in electrical engineering from Xian Jiaotong University, Xian, China, in 1991 and 1994, respectively, and the Ph.D. degree in electronics and computer engineering from Brunel University, Uxbridge, U.K., in 2001. He is currently a Professor with the School of Advanced Technology and acting the Head of Department of Mechatronics and Robotics, Xi'an Jiaotong-Liverpool University (XJTLU), Suzhou 215123, China. Prior to joining XJTLU, he was a professor with different universities in the UK. He is a general chair/technical programme chair for CollaborateCom over the last 5 years and a guest editor for a number of international journals. $\mathrm{He}$ has been an Investigator or Co-Investigator of nearly 30 research projects sponsored from EU, UK, EPSRC, Innovate UK, China NSFC, and industry. The research has led to the publications of over 180 referred papers, 15 granted patents, and development of a few commercial products in condition monitoring, wireless mesh networks, and user-centric routing and navigation for group users. The smart trolley he has developed with an industry partner is the first airport Internet of Things (IoT) product in the world to provide smart services to air passengers. His current research interests lie in industrial IoT, edge AI for industrial machine vision, indoor positioning, acoustic localization, communications and sensing, and personalized navigation and services for group travellers.

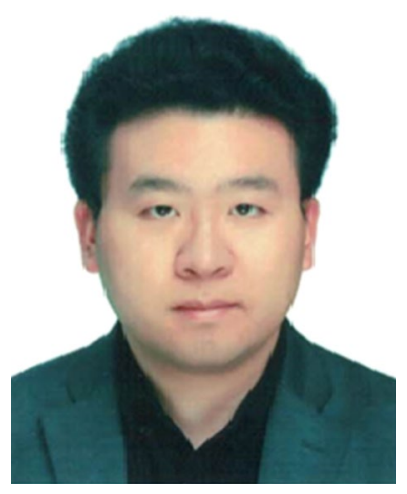

Honghao Gao received a Ph.D. in Computer Science and started his academic career at Shanghai University in 2012. He is currently with the School of Computer Engineering and Science, Shanghai University, China. He is also a professor at Gachon University, South Korea. Prior to that, he was a research fellow at the Software Engineering Information Technology Institute at Central Michigan University (CMU), USA and was an adjunct professor at Hangzhou Dianzi University, China. His research interests include formal software verification, industrial IoT/wireless networks, service collaborative computing, and intelligent medical image processing. He has publications in IEEE TII, IEEE T-ITS, IEEE 
IoT-J, IEEE TNSE, IEEE TCCN, IEEE TGCN, IEEE/ACM TCBB, ACM TOIT, ACM TOMM, ACM TMIS, IEEE TCSS, IEEE TETCI, IEEE JBHI, and IEEE Network. He is a fellow of IET, BCS, and EAI and a senior member of IEEE, CCF, and CAAI. He is the Editor-inChief of the International Journal of Intelligent Internet of Things Computing (IJIITC), Editor of Wireless Network (WINE) and IET Wireless Sensor Systems (IET WSS), and Associate Editor for IEEE Transactions on Intelligent Transportation Systems (IEEE T-ITS), IET Software, International Journal of Communication Systems (IJCS), and Journal of Internet Technology (JIT). Moreover, he has broad working experience in cooperative industry-university research. He is a European Union Institutions-appointed external expert for reviewing and monitoring the EU Project, is a member of the EPSRC Peer Review Associate College for UK Research and Innovation in the UK, and is also a founding member of the IEEE Computer Society Smart Manufacturing Standards Committee.

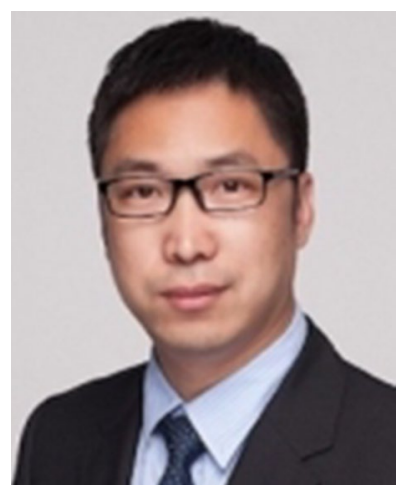

Kaizhu Huang is currently a Professor with Department of Intelligent Science, and the Associate Dean of Research and Graduate Studies, School of Advanced Technology, Xi'an JiaotongLiverpool University (XJTLU). Prof. Huang obtained the B.E. in automatic control from Xi'an Jiaotong University, in 1997, the M.E from Institution of Automation, Chinese Academy of Sciences (CAS) in 2000, and the Ph.D. degree from Chinese University of Hong Kong (CUHK) in 2004. He worked in Fujitsu Research Centre, CUHK, University of Bristol, National Laboratory of Pattern Recognition, CAS from 2004 to 2012. Prof. Huang has been working in machine learning, neural information processing, and pattern recognition. He was the recipient of 2011 Asia Pacific Neural Network Society Young Researcher Award. He received best paper or book award six times. He has authored or co-authored 9 books and over 200 international research papers (80+ international journals) e.g., in journals (JMLR, Neural Computation, IEEE T-PAMI, IEEE T-NNLS, IEEE T-BME, IEEE T-Cybernetics) and conferences (NeurIPS, IJCAI, SIGIR, UAI, CIKM, ICDM, ICML, ECML, CVPR). He serves as associated editors/advisory board members in a number of journals and book series. He was invited as keynote speaker in more than 20 international conferences or workshops. 